\title{
Minimally invasive surgery for left ventricular assist device implantation is safe and associated with a decreased risk of right ventricular failure
}

\author{
Adrien Carmona ${ }^{1,2}$, Tam Hoang Minh ${ }^{1}$, Stéphanie Perrier ${ }^{1}$, Clément Schneider $^{1}$, Sandrine Marguerite ${ }^{3}$, \\ Gharib Ajob $^{3}$, Cristinar Mircea ${ }^{3}$, Paul-Michel Mertes ${ }^{3}$, Darmesh Ramlugun ${ }^{1}$, Joseph Atlan ${ }^{1}$, \\ Jean-Jacques Von Hunolstein ${ }^{2}$, Eric Epailly ${ }^{1}$, Jean-Philippe Mazzucotelli ${ }^{1}$, Michel Kindo ${ }^{1}$ \\ ${ }^{1}$ Department of Cardiovascular Surgery, ${ }^{2}$ Department of Cardiology, ${ }^{3}$ Department of Anesthesia and Intensive Care Unit, University Hospitals of \\ Strasbourg, Strasbourg, France \\ Contributions: (I) Conception and design: All authors; (II) Administrative support: M Kindo; (III) Provision of study materials or patients: S Perrier, \\ T Hoang Minh, C Schneider, JJ Von Hunolstein, E Epailly, JP Mazzucotelli, M Kindo; (IV) Collection and assembly of data: S Perrier, T Hoang \\ Minh, C Schneider, S Marguerite, G Ajob, C Mircea, JJ Von Hunolstein, E Epailly, JP Mazzucotelli, M Kindo; (V) Data analysis and interpretation: \\ A Carmona, S Perrier, T Hoang Minh, C Schneider, JJ Von Hunolstein, E Epailly, JP Mazzucotelli, M Kindo; (VI) Manuscript writing: All authors; \\ (VII) Final approval of manuscript: All authors. \\ Correspondence to: Professor Michel Kindo, MD, PhD. Service de Chirurgie Cardiovasculaire, NHC, 1 place de l'Hôpital, BP 426-67091 Strasbourg \\ Cedex, France. Email: michel.kindo@chru-strasbourg.fr.
}

Background: Right ventricular failure (RVF) after left ventricular assist device (LVAD) implantation is associated with significant mortality and morbidity. The objective of this study was to determine pre- and postoperative risk factors associated with the occurrence of RVF after LVAD implantation.

Methods: This retrospective study included 68 patients who received LVADs between 2010 and 2018 either for bridge to transplant (40 patients, 58.8\%) or bridge to destination therapy (28 patients, $41.2 \%$ ). RVF after LVAD implantation was defined according to the INTERMACS classification. The primary endpoint was the occurrence of RVF. The secondary endpoints were hospital mortality and morbidity and long-term survival.

Results: The majority of patients (61.8\%) had an INTERMACS profile 1 (36.8\%) or $2(25.0 \%)$. The LVAD was implanted either by sternotomy (37 patients, $54.4 \%$ ) or thoracotomy (31 patients, $45.6 \%$ ). RVF after LVAD implantation was observed in 32 patients (47.1\%). In univariate analysis, an elevated serum glutamic oxaloacetic transaminase (SGOT) $(\mathrm{P}=0.028)$ and a high preoperative vasoactive inotropic score (VIS) ( $\mathrm{P}=0.028)$ were significantly associated with an increased risk of RVF, whereas the implantation of LVAD through a thoracotomy approach was associated with a significant reduction in this risk $(\mathrm{P}=0.006)$. The multivariate analysis demonstrated that only the thoracotomy approach was significantly associated with decreased risk of RVF (odds ratio $=0.33,95 \%$ confidence interval: $0.17-0.96 ; \mathrm{P}=0.042$ ). Hospital mortality was $53.1 \%$ and $5.6 \%$ in the RVF and control groups, respectively $(\mathrm{P}<0.0001)$. The incidence of stroke and postoperative acute renal failure were significantly increased in the RVF group compared with the control group. The survival after LVAD implantation was $33.5 \% \pm 9.0 \%$ and $85.4 \% \pm 6.0 \%$ at 1 year in the RVF and control groups, respectively $(\mathrm{P}<0.0001)$.

Conclusions: LVAD implantation by thoracotomy significantly reduced the risk of postoperative RVF. This surgical approach should, therefore, be favored.

Keywords: Heart failure (HF); artificial heart ventricle; care, postoperative

Submitted Sep 28, 2019. Accepted for publication Jan 17, 2020.

doi: $10.21037 /$ jtd.2020.02.32

View this article at: http://dx.doi.org/10.21037/jtd.2020.02.32 


\section{Introduction}

The prevalence of severe end-stage heart failure (HF) is increasing worldwide. Despite progress in medical therapy, some patients with end-stage HF will need left ventricular assist device (LVAD) implantation as a bridge to transplant (BTT) or destination therapy (DT) to improve survival and quality of life (1).

Right ventricular failure (RVF) after LVAD implantation remains a major complication with a prevalence ranging between $9-51 \%$ and is significantly associated mortality and morbidity $(2,3)$. For that reason, clinicians evaluating patients for LVAD implantation must be aware of the risk factors for RVF after LVAD implantation $(2,3)$. Several preoperative clinical, biological, hemodynamic or echocardiographic variables have been assessed as risk factors for post-LVAD RVF, but none of these risk factors allow optimal patient selection (2,3). This situation supports a multidisciplinary approach for patient selection and the development of perioperative strategies to prevent RVF. Furthermore, several changes have occurred in the last decade with the switch from an axial pump to a smaller centrifugal pump and the development of a less invasive approach for LVAD implantation through a thoracotomy $(4,5)$. These changes may have an impact on RVF prevalence.

The aim of this study was to determine the risk factors for RVF after LVAD implantation and to evaluate the impact of RVF on mortality and morbidity.

\section{Methods}

\section{Study population and data collection}

A retrospective review of all consecutive patients undergoing LVAD implantation in our institution between January 1, 2010, and May 1, 2018, was performed. Sixty-eight patients were enrolled in this single-center, observational study. For all consecutive patients operated in our department, preoperative, intraoperative and postoperative data were systematically and prospectively collected in a database (ASTR, Access, Microsoft ${ }^{\circledR}$ ). Because, the study is a retrospective analysis of institutional data, and in compliance with the French law on clinical research, we only had to obtain French personal data protection authorization. We submitted our study to the national commission for data protection (CNIL) and obtained authorization. The study was approved by institutional ethic committee of the University Hospitals of Strasbourg.

\section{Operative approach}

The thoracotomy approach was started in 2016 and included two approaches: mini-sternotomy plus left anterolateral thoracotomy and left posterolateral thoracotomy. Our present surgical strategy regarding the surgical approach for LVAD implantation is reported in Figure 1.

For the mini-sternotomy plus left anterolateral thoracotomy approach, we followed the surgical technique described elsewhere (6). In our practice, an upper hemisternotomy with a "T inverted" incision was performed. The left ventricular apex was systematically located with a transthoracic echography, and the left thoracotomy incision was performed facing the apex. When cardiopulmonary bypass was utilized, central cannulation was favored. The sewing ring was attached, and after initiating the cardiopulmonary bypass, the left ventricle was cored, and the inflow cannula was introduced and fixed to the ring. The outflow graft was placed based on the lateral wall of the left ventricle in front of the pulmonary artery, and an endto-side anastomosis was performed on the ascending aorta.

For the left posterolateral approach, the left ventricular apex was located with the transthoracic echocardiography, and the thoracotomy was performed facing the apex. The sewing ring was placed first and after heparinization, and the outflow graft was anastomosed end-to-side on the descending aorta using a partial cross-clamp. The femoral vessels were used, and cardiopulmonary bypass was started. The left ventricle was cored, and the inflow cannula was introduced after retrograde de-airing and fixed to the ring.

Since 2017 , an epicardial echocardiography was systematically used to determine the best inflow cannula placement of the LVAD to ensure the absence of conflict with the left ventricular wall; that the inflow cannula will be parallel the interventricular septum and coaxial with the mitral orifice and to eliminate any trabeculation or thrombus.

\section{Study endpoints}

Right ventricular (RV) dysfunction after LVAD implantation was stratified by the Mechanically Assisted Circulatory Support (INTERMACS) classification. Three levels of $\mathrm{RV}$ dysfunction were defined by the duration of inotrope support [mild ( $\leq 7$ days), moderate (8-14 days) and severe ( $>14$ days)] or the implantation of a mechanical RV assist device (3).

The primary study endpoint was RVF, which was 


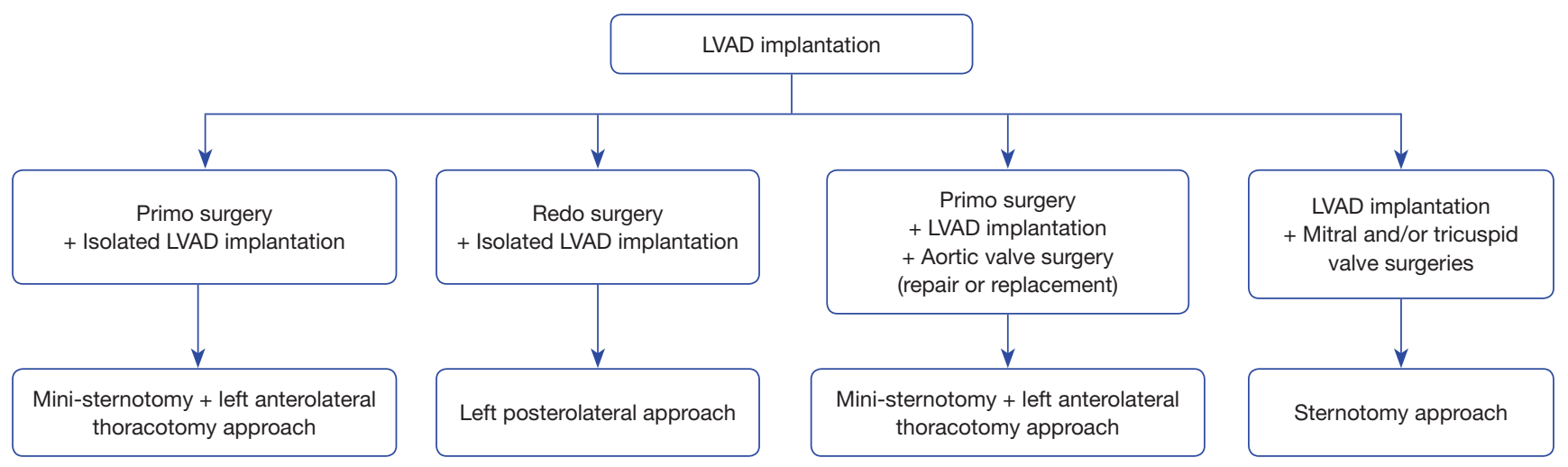

Figure 1 Surgical approach strategy for LVAD implantation. LVAD, left ventricular assist device.

defined as moderate or severe RV dysfunction by the INTERMACS classification (3). Of the 68 patients enrolled in this study, 32 (47.1\%) developed RVF. Twelve patients (17.6\%) and 20 patients (29.4\%) had moderate or severe RV dysfunction, respectively. Among the patients with severe RV dysfunction, 13 (19.1\%) patients had a temporary RV assist device.

The secondary endpoints were hospital mortality and morbidity and long-term survival.

\section{Statistical analysis}

Two groups were defined: an RVF group and a control group. The data are presented as the mean \pm standard deviation (SD). Differences between the categorical variables were tested using chi-square or Fisher' exact tests depending on the expected values. Differences between continuous variables were tested using Student's $t$-tests or the Mann-Whitney U-tests depending on whether there was a Gaussian distribution.

Potential risk factors for RVF (RVF group) were first tested using a univariate analysis. After performing the univariate analysis, only the variables with a $\mathrm{P}$ value less than 0.05 , which were determined using a two-tailed $t$-test, were used in the multivariate logistic regression analysis (with a backward, stepwise method based on the likelihood ratio test). The odds ratios and their corresponding $95 \%$ confidence intervals are reported in addition to their associated $\mathrm{P}$ values.

The cumulative probability of survival was estimated with the Kaplan-Meier method and was compared between groups using a log-rank test.

The statistical computations were performed using SPSS
$\left(\mathrm{SPSS}^{\circledR}\right.$, version 17.0, Chicago, IL, USA).

\section{Results}

\section{Preoperative characteristics}

The preoperative variables sorted by occurrence of RVF are reported in Tables 1,2 .

The overall mean age was $59.6 \pm 13.4$ years. Most patients (61.8\%) were categorized as either INTERMACS Profile 1 (36.8\%) or Profile 2 (25.0\%). The indications for LVAD implantation were bridge-to-transplant for 40 patients (58.8\%) and DT for 28 patients (41.2\%).

The patient demographics and echography parameters were similar in the two groups (Table 1). Only serum glutamic oxaloacetic transaminase (SGOT) was significantly higher in the RVF group compared to the control group $(\mathrm{P}=0.028)$.

Preoperative short-term mechanical support was used in $33.8 \%$ of the patients with no differences between the 2 groups, including no differences in the type of shortterm mechanical support and the length of preoperative support (Table 1). The preoperative mean arterial pressure, central venous pressure, cardiac index and central venous oxygen saturation were similar between the two groups. The vasoactive inotropic score (VIS) was calculated using the equation described elsewhere (7): VIS $=$ dopamine dose $(\mu \mathrm{g} / \mathrm{kg} / \mathrm{min})+$ dobutamine dose $(\mu \mathrm{g} / \mathrm{kg} / \mathrm{min})+100 \times$ epinephrine dose $(\mu \mathrm{g} / \mathrm{kg} / \mathrm{min})+10 \times$ milrinone dose $(\mu \mathrm{g} / \mathrm{kg} / \mathrm{min})+10,000 \times$ vasopressin dose $($ units $/ \mathrm{kg} / \mathrm{min})+$ $100 \times$ norepinephrine dose $(\mu \mathrm{g} / \mathrm{kg} / \mathrm{min})$. The VIS was significantly higher in the RVF group than in the control group $(\mathrm{P}=0.028$, Table 2$)$. 
Table 1 Preoperative characteristics of the study cohorts

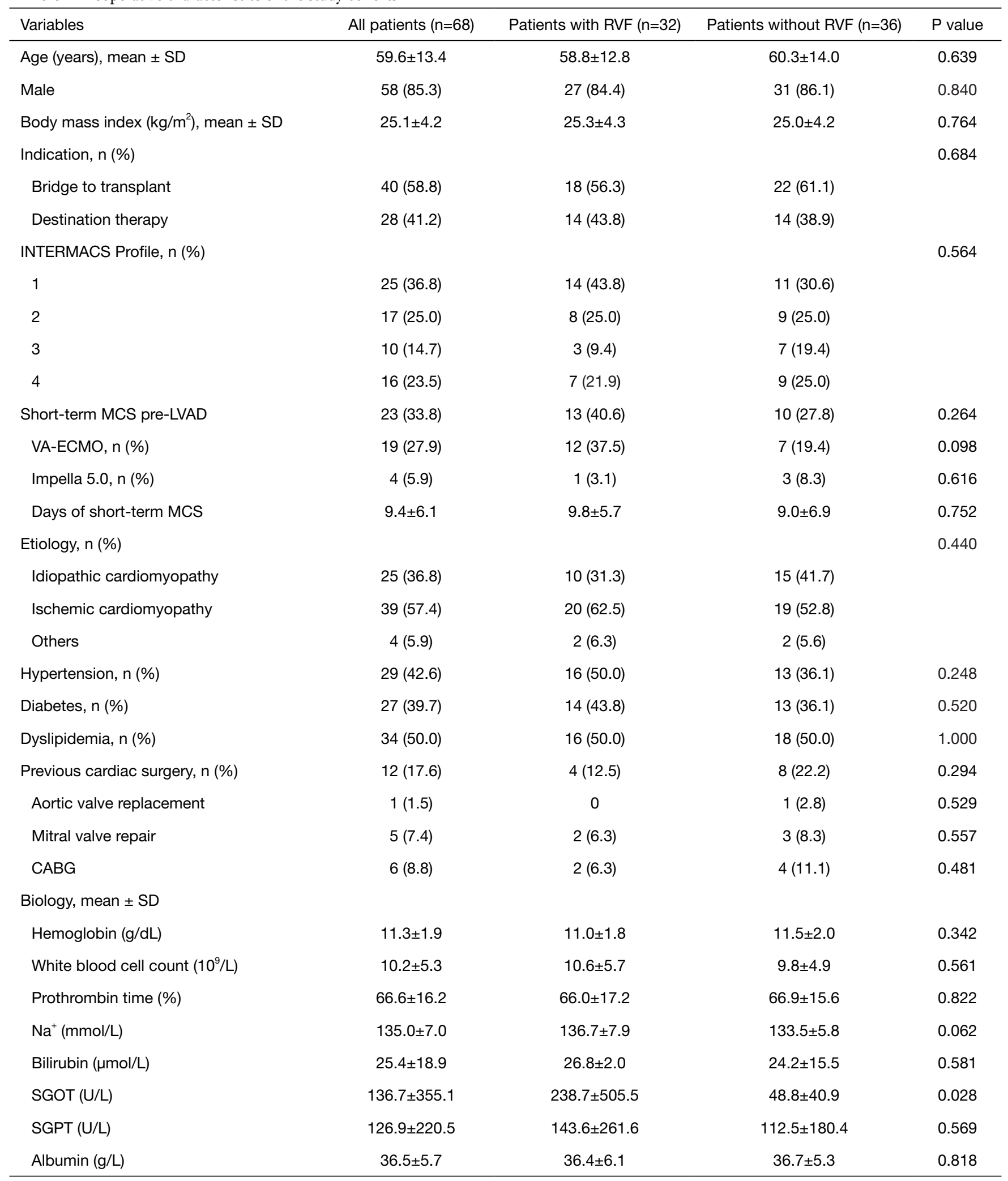

Table 1 (continued) 
Table 1 (continued)

\begin{tabular}{|c|c|c|c|c|}
\hline Variables & All patients $(n=68)$ & Patients with RVF $(n=32)$ & Patients without RVF (n=36) & $P$ value \\
\hline Troponin I ( $\mu \mathrm{g} / \mathrm{L})$ & $113.6 \pm 384.6$ & $101.3 \pm 257.4$ & $124.1 \pm 471.7$ & 0.837 \\
\hline Lactates (mmol/L) & $2.2 \pm 7.9$ & $3.3 \pm 11.2$ & $1.1 \pm 0.3$ & 0.247 \\
\hline \multicolumn{5}{|l|}{ Echography, mean \pm SD or $n(\%)$} \\
\hline LV mass/BSA $\left(\mathrm{g} / \mathrm{m}^{2}\right)$ & $149.3 \pm 49.8$ & $144.7 \pm 46.2$ & $152.7 \pm 53.1$ & 0.625 \\
\hline LVEDD (mm) & $69.2 \pm 10.3$ & $67.5 \pm 9.6$ & $70.5 \pm 10.8$ & 0.276 \\
\hline LVESD (mm) & $60.8 \pm 11.0$ & $58.5 \pm 10.7$ & $62.2 \pm 11.1$ & 0.293 \\
\hline E/A ratio & $2.0 \pm 1.0$ & $2.1 \pm 1.1$ & $1.9 \pm 0.9$ & 0.659 \\
\hline Right atrial area $\left(\mathrm{cm}^{2}\right)$ & $25.3 \pm 7.0$ & $25.9 \pm 6.6$ & $25.0 \pm 7.4$ & 0.746 \\
\hline RVFAC (\%) & $28.5 \pm 11.7$ & $29.0 \pm 13.3$ & $28.2 \pm 10.9$ & 0.824 \\
\hline S'-wave DTI (cm/s) & $8.9 \pm 3.4$ & $9.2 \pm 3.5$ & $8.8 \pm 3.3$ & 0.701 \\
\hline TAPSE (mm) & $14.4 \pm 6.2$ & $13.8 \pm 6.9$ & $14.7 \pm 6.0$ & 0.713 \\
\hline TR grade $\geq 3$ & 0 & 0 & 0 & 1.000 \\
\hline MR grade $\geq 3, n(\%)$ & $14(20.6)$ & $5(15.6)$ & $9(25.0)$ & 0.340 \\
\hline sPAP (mmHg) & $48.4 \pm 13.6$ & $49.8 \pm 13.4$ & $47.4 \pm 13.9$ & 0.559 \\
\hline
\end{tabular}

RVF, right ventricular failure; MCS, mechanical support; LVAD, left ventricular assist device; VA-ECMO, veno-arterial extracorporal membrane oxygenation; CABG, coronary artery bypass grafting; SGOT, serum glutamic oxaloacetic transaminase; SGPT, serum glutamic-pyruvic transaminase; GFR, glomerular filtration rate, as determined with the Modification of Diet in Renal Disease (MDRD) study equation; LVEF, left ventricular ejection fraction; LV, left ventricular; BSA, body surface area; LVEDD, LV end-diastolic diameter; LVESD, LV end-systolic diameter; RVFAC, right ventricular fractional area change; S'-wave DTI, Doppler tissue imaging (DTI)-derived tricuspid lateral annular systolic; TAPSE, tricuspid annular plane systolic excursion; TR, tricuspid regurgitation grade; MR, mitral regurgitation grade; sPAP, systolic pulmonary artery pressure; BSA, body surface area; E/A ratio, ratio of mitral E velocity to mitral $A$ velocity.

\section{Operative characteristics}

The operative data are reported in the Table 3.

The LVAD implanted was the Heartmate II in 15 patients $(22.1 \%)$, the HeartWare ventricular assist device (HVAD) in 52 patients (76.5\%) and the Heartmate III in 1 patient $(1.5 \%)$.

The LVAD implantation was performed through a median sternotomy in 37 patients $(54.4 \%)$ and through a thoracotomy approach in 31 patients $(45.6 \%)$. The median sternotomy approach was used significantly more often in the RVF group (71.9\%) than in the control group (38.9\%; $\mathrm{P}=0.006$; Table 3). Four patients in the control group with preoperative extracorporeal membrane oxygenation (ECMO) underwent LVAD implantation under only
ECMO support, and 1 patient was off the pump.

\section{Risk factors for RVF after LVAD implantation}

The preoperative and operative patient characteristics (with a $P$ value less than 0.05 in the univariate analysis) of the control and RVF groups are reported in Tables 1-3.

Univariate analysis revealed significant associations between RVF and the preoperative SGOT level $(\mathrm{P}=0.028)$, preoperative VIS $(\mathrm{P}=0.028)$ and thoracotomy approach $(\mathrm{P}=0.006)$.

In multivariate logistic regression analysis predicting RVF after LVAD implantation, only the thoracotomy approach was significantly associated with a decreased risk 
Table 2 Preoperative hemodynamic status

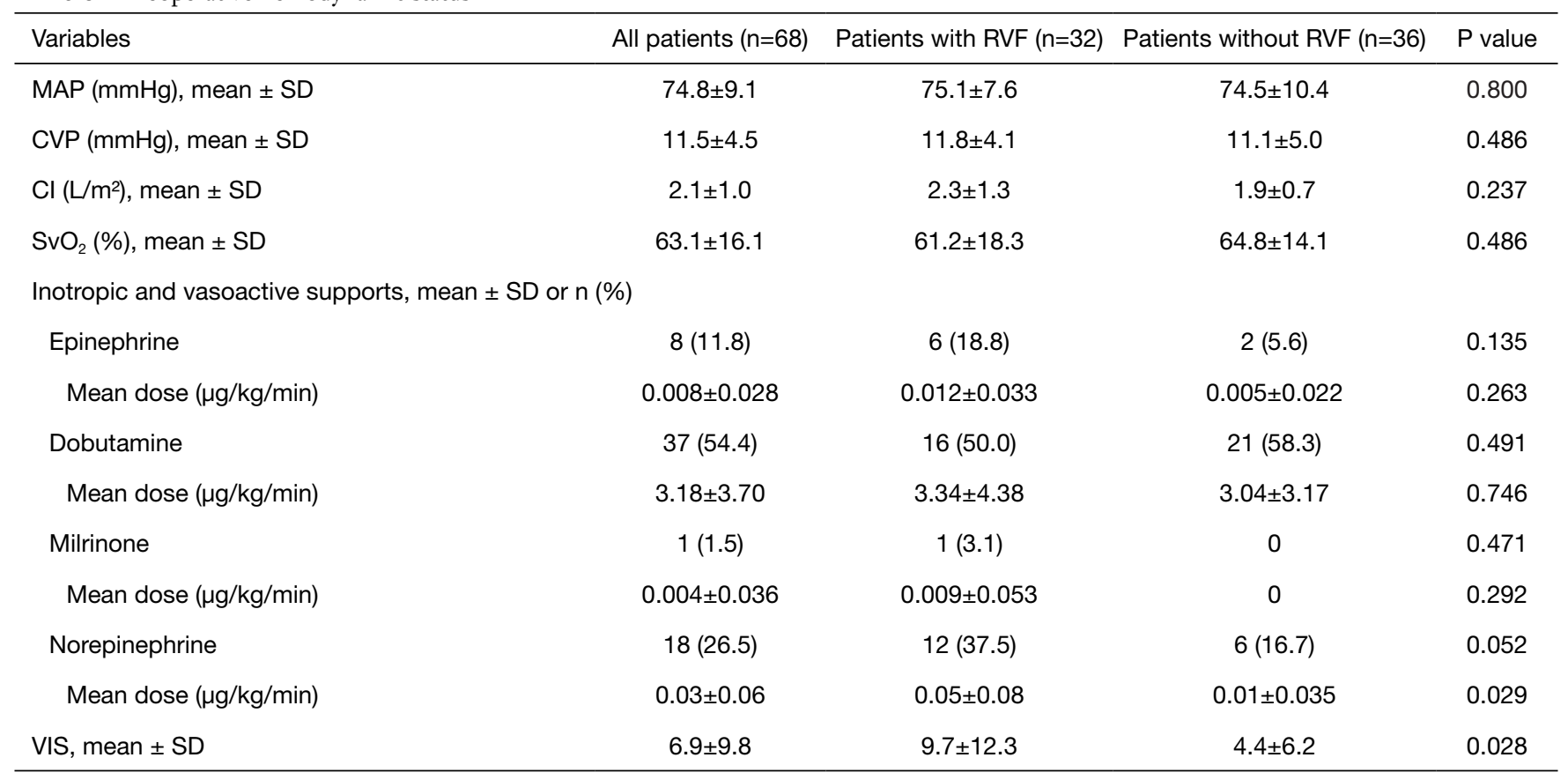

RVF, right ventricular failure; MAP, mean arterial pressure; CVP, central venous pressure; $\mathrm{Cl}$, cardiac index; $\mathrm{SvO}_{2}$, central venous oxygen saturation; VIS, vasoactive-inotropic score [VIS = dopamine dose $(\mu \mathrm{g} / \mathrm{kg} / \mathrm{min})+$ dobutamine dose $(\mu \mathrm{g} / \mathrm{kg} / \mathrm{min})+100 \times$ epinephrine dose $(\mu \mathrm{g} / \mathrm{kg} / \mathrm{min})+10 \times$ milrinone dose $(\mu \mathrm{g} / \mathrm{kg} / \mathrm{min})+10,000 \times$ vasopressin dose (units $/ \mathrm{kg} / \mathrm{min})+100 \times$ norepinephrine dose $(\mu \mathrm{g} / \mathrm{kg} / \mathrm{min})]$.

Table 3 Operative characteristics

\begin{tabular}{|c|c|c|c|c|}
\hline Variables & All patients $(n=68)$ & Patients with RVF ( $n=32)$ & Patients without RVF $(n=36)$ & $P$ value \\
\hline Thoracotomy approach, n (\%) & $31(45.6)$ & $9(28.1)$ & $22(61.1)$ & 0.006 \\
\hline $\begin{array}{l}\text { Left thoracotomy with anastomosis of the OG } \\
\text { to the descending aorta }\end{array}$ & $13(19.1)$ & $3(9.4)$ & $10(27.8)$ & 0.069 \\
\hline Implantation under ECMO, n (\%) & $4(5.9)$ & 0 & $4(11.1)$ & 0.116 \\
\hline Off pump, n (\%) & $1(1.5)$ & 0 & $1(2.8)$ & 1.000 \\
\hline CPB time (minutes), mean \pm SD & $117.6 \pm 49.1$ & $129.3 \pm 53.8$ & $105.6 \pm 41.2$ & 0.055 \\
\hline Associated procedure, $\mathrm{n}(\%)$ & $2(2.9)$ & $1(3.1)$ & $1(2.8)$ & 1.000 \\
\hline
\end{tabular}

RVF, right ventricular failure; OG, outflow graft of left ventricular assist device; ECMO, veno-arterial extracorporal membrane oxygenation; CPB, cardiopulmonary bypass. 


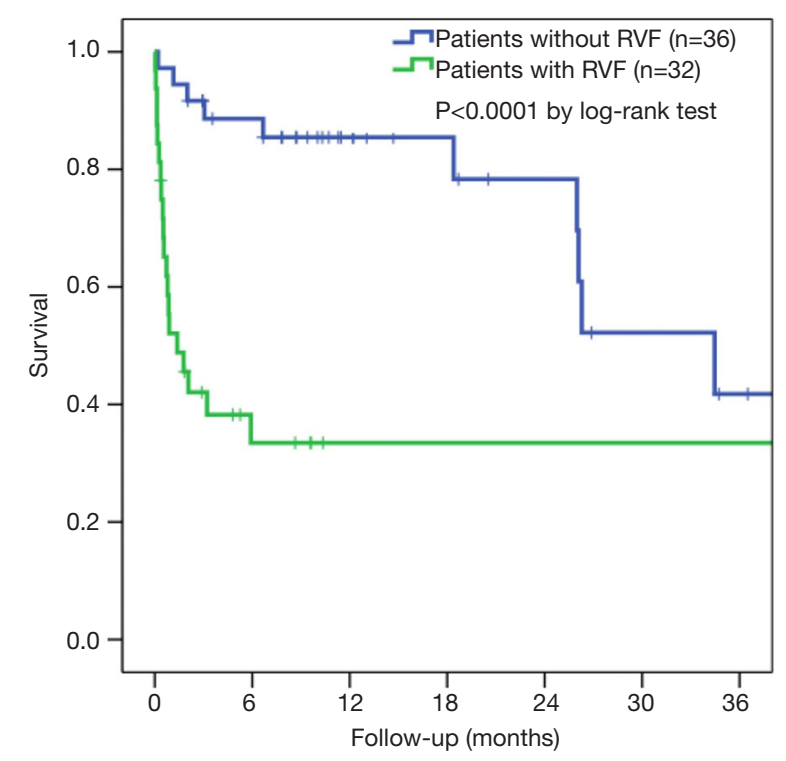

\begin{tabular}{|l|c|c|c|c|c|c|c|}
\hline Follow-up (months) & 0 & 6 & 12 & 18 & 24 & 30 & 36 \\
\hline No. at risk: & & & & & & & \\
\hline Patients without RVF & 36 & 28 & 16 & 12 & 9 & 5 & 3 \\
\hline Patients with RVF & 32 & 7 & 3 & 3 & 3 & 3 & 3 \\
\hline
\end{tabular}

Figure 2 Three-year survival (Kaplan-Meier analysis). RVF, right ventricular failure.

of RVF (odds ratio $=0.33,95 \%$ confidence interval: $0.17-$ $0.96 ; \mathrm{P}=0.042)$.

\section{Hospital mortality and survival}

Table 4 shows early postoperative outcomes and long-term survival.

The overall in-hospital mortality was $27.9 \%$. RVF was associated with increased in-hospital mortality (53.1\% versus $5.6 \%, \mathrm{P}<0.0001)$. The mean survival censored for transplantation was significantly better for patients without RVF than those with RVF ( $46.7 \pm 8.4$ versus $19.6 \pm 5.0$ months, respectively; $\mathrm{P}<0.0001)$. The overall survival rates were $60.7 \% \pm 9.2 \%, 52.7 \% \pm 9.6 \%$ and $26.0 \% \pm 10.5 \%$ at 6 months, 1 year and 2 years, respectively. The survival rates in the RVF and control groups at 1 and 2 years were $28.6 \% \pm 12.1 \%$ versus $85.7 \% \pm 9.4 \%$ and $19.0 \% \pm 11.2 \%$ versus $41.1 \% \pm 14.8 \%$, respectively $(\mathrm{P}<0.0001$ by log-rank test, Figure 2).

There were 13 (19.1\%) patients who required RV assist device implantation. Among these patients, six patients died, and seven patients were successfully weaned from their temporary mechanical support after a mean support time of $10.5 \pm 5.6$ days.

No significant differences were found regarding mean arterial pressure, central venous pressure or cardiac index at admission to the intensive care or on the first postoperative day. The VIS and lactate level on the first postoperative day were significantly higher in the RVF group than the control group. The incidence of permanent stroke was significantly increased in the RVF group compared to the control group (21.9\% versus $2.8 \%$, respectively; $\mathrm{P}=0.022$ ). A total of 36 (52.9\%) patients needed postoperative renal replacement therapy with a higher incidence in the RVF group than in the control group ( $\mathrm{P}=0.003$; Table 4). Mechanical ventilation time was significantly higher in the RVF group than in the control group $(\mathrm{P}=0.005)$, whereas the intensive care unit and hospital lengths of stay did not differ between the 2 groups.

\section{Discussion}

The aim of this study was to determine the risk factors for RVF after LVAD implantation. The main results of the 
Table 4 Postoperative outcomes

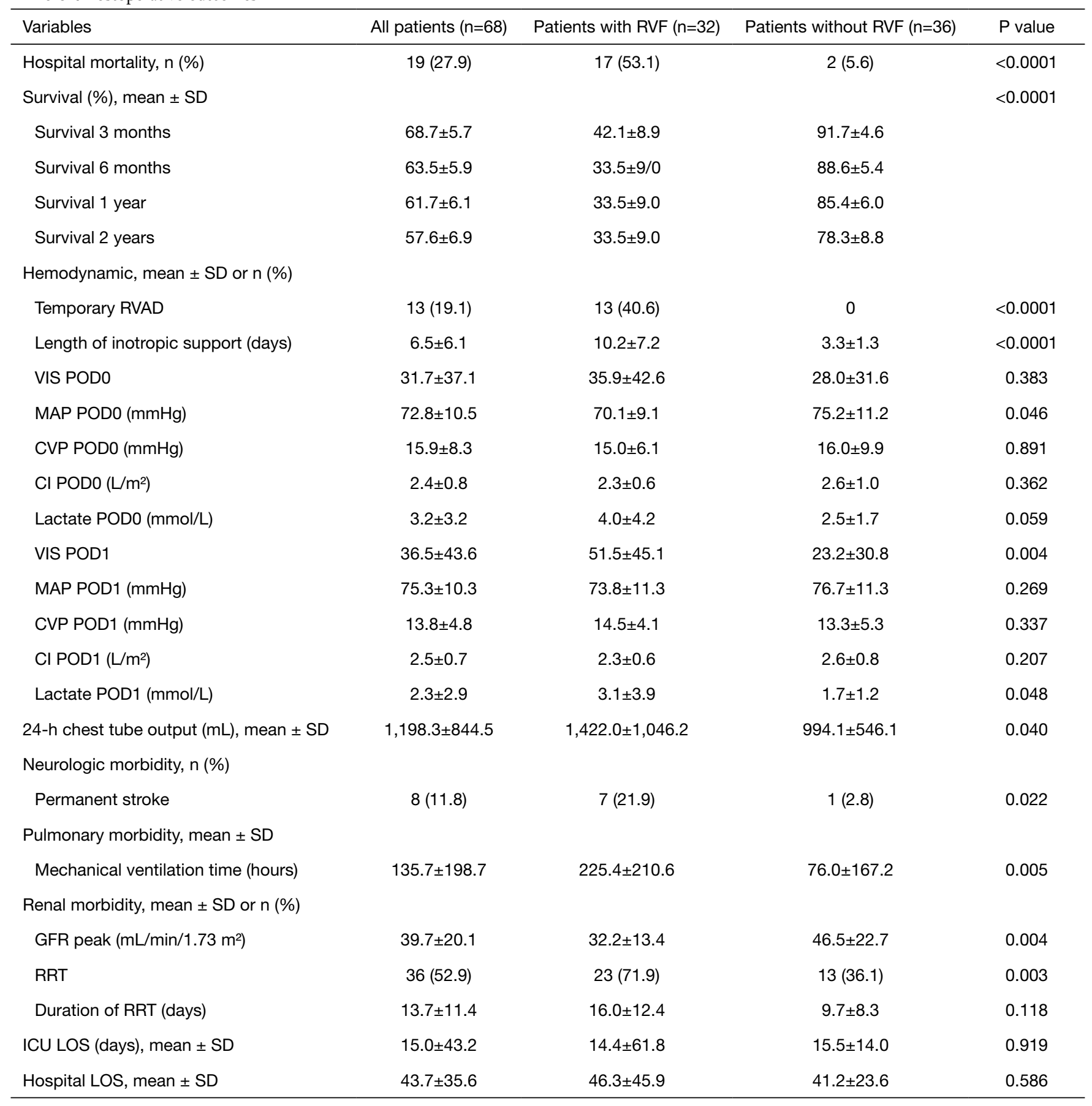

RVF, right ventricular failure; RVAD, right ventricular assist device; VIS, vasoactive-inotropic score [VIS = dopamine dose $(\mu \mathrm{g} / \mathrm{kg} / \mathrm{min})$ + dobutamine dose $(\mu \mathrm{g} / \mathrm{kg} / \mathrm{min})+100 \times$ epinephrine dose $(\mu \mathrm{g} / \mathrm{kg} / \mathrm{min})+10 \times$ milrinone dose $(\mu \mathrm{g} / \mathrm{kg} / \mathrm{min})+10,000 \times$ vasopressin dose (units $/ \mathrm{kg} / \mathrm{min})+100 \times$ norepinephrine dose $(\mu \mathrm{g} / \mathrm{kg} / \mathrm{min})]$; POD, postoperative day; MAP, mean arterial pressure; CVP, central venous pressure; $\mathrm{Cl}$, cardiac index; GFR, glomerular filtration rate, as determined with the Modification of Diet in Renal Disease (MDRD) study equation; RRT, renal replacement therapy; LOS, length of stay. 
study are as follow: (I) the thoracotomy approach for LVAD implantation significantly reduces the risk of postoperative RVF in our population with a high preoperative risk profile; (II) RVF significantly impacts hospital mortality, long-term survival and hospital morbidity.

Minimally invasive techniques (mini-sternotomy and/or mini-thoracotomy) for cardiac surgery are now increasingly used to avoid the full sternotomy approach and, thus, the specific complications associated with the sternotomy (8). However, to date, there is no clear evidence that a minimally invasive approach for cardiac surgery is superior or inferior to the full sternotomy approach in outcomes, especially because of the longer cross-clamp and cardiopulmonary bypass times associated with minimally invasive approaches (8). Schmitto et al. were the first to describe a minimally approach for LVAD implantation $(6,9)$ using a left mini-thoracotomy and a J-shaped hemisternotomy $(6,9)$. This less invasive technique for LVAD implantation is increasingly used worldwide, and there is now growing evidence of its benefits compared to the full sternotomy approach (10-15). These studies have demonstrated the feasibility of this technique.

We found that the thoracotomy approach was associated with a significant decrease in RVF risk after LVAD implantation. Other studies reported that less invasive approaches were associated with a significant reduction in RVF incidence (13-15). Our study is the first to demonstrate the strong positive impact of the thoracotomy approach to prevent RVF with a multivariate risk model analysis. We started this strategy in 2016, and it is now the reference technique for LVAD implantation. Even in the most severe profiles, this technique may be used with a significant benefit in the risk of RVF, as others have also demonstrated (13).

The protective effect of the thoracotomy approach on the risk of RVF may be explained by three mechanisms: the preservation of pericardium function, the absence of twisting the right ventricle, and the reduction in cardiopulmonary bypass time. The pericardium serves important mechanical functions as a constraint to ventricular filling and ventricular interaction (16). During LVAD implantation with a thoracotomy approach, the pericardium of the right ventricle remains intact and, for that reason, it may preserve RV function (17). Contrary to the full sternotomy approach, with the minimally invasive technique, the heart remains in place and the pyramidal shape of the right ventricle is preserved. Indeed, the modification of this shape impacts its filling and performance (18). Finally, minimally invasive LVAD implantation is performed with shorter cardiopulmonary bypass time, which directly impacts the incidence of postoperative blood loss and red blood cell transfusions that are well known to be associated with the risk of RVF $(11,13,14,19)$. With the univariate analysis, we observed that the preoperative VIS was associated with an increased risk of postoperative RVF. However, this factor was not associated with this risk in the multivariate analysis, possibly due to our small sample size. Nevertheless, we believe that the VIS must incorporate an overall assessment of the patient before LVAD implantation with particular emphasis on the vasoconstrictive part of this score.

We believe that the thoracotomy approach for LVAD implantation is the only truly less invasive technique compared to the other minimally invasive techniques used in cardiac surgery. Indeed, we have easy direct access to the left ventricular apex and ascending aorta for placement of the LVAD, and the surgery can be performed off-pump or with a significant reduction in cardiopulmonary bypass time compared to the full sternotomy approach $(11,13,14)$.

The incidence of RVF ranges from $9 \%$ to $51 \%$ in the literature (2,3). An explanation for this significant variance may be related to the definition of right ventricular hypertrophy (RVH) used, the preoperative patient characteristics, the indication (BTT or DT) and the type of LVAD implanted (pulsatile flow or continuous flow) $(2,3)$. When comparing the preoperative characteristics of our population to the EUROMACS registry, our patients were older, more frequently categorized as INTERMACS profiles 1 and 2 and more often received a DT device strategy (20). These differences explain our prevalence of RVF and that approximately one-third of our patients were on preoperative, short-term, mechanical support.

Our results confirmed prior studies of the impact of RVF after LVAD implantation on hospital mortality and long-term survival $(2,3,19)$. In a recent study, Han et al. demonstrated that early postoperative VIS is a significant predictor of hospital mortality with an odds ratio of 1.06 per unit increase in VIS (7). These results are in good agreement with our study. Indeed, the VIS was significantly increased in the RVF group compared to the control group on the first postoperative day and was related to a significant increase in the lactate level. Several studies have demonstrated, as in our study, that patients with RVF are at risk of multiorgan dysfunction and acute kidney injury $(19,21)$. Our study confirmed that, in patients presenting with RVF after LVAD implantation, stroke incidence is significantly increased (19). This finding may be explained by the need to support the failing right ventricle with a 
temporary mechanical support, which makes management of postoperative haemostasis particularly difficult.

\section{Limitations}

This study had several limitations. This study was a retrospective, single-center, observational study that compared two surgical techniques performed consecutively without randomization. Our results will need to be confirmed with a prospective, multicenter, randomized study. We had a small sample size that included mostly critically ill patients. A larger sample size would have been powered for the statistical analysis.

\section{Conclusions}

This study demonstrates that the thoracotomy approach significantly decreased the risk of RVF after LVAD implantation. Due to the high in-hospital mortality, the negative impact on long-term survival and the increased morbidity associated with RVF, the thoracotomy approach should be the reference technique for LVAD implantation.

\section{Acknowledgments}

We thank Mrs. Marie Tyman Heinrich for her help in collecting data.

Funding: None.

\section{Footnote}

Conflicts of Interest: All authors have completed the ICMJE uniform disclosure form (available at http://dx.doi. org/10.21037/jtd.2020.02.32). The authors have no conflicts of interest to declare.

Ethical Statement: The authors are accountable for all aspects of the work to ensure that questions related to the accuracy or integrity of any part of the work are appropriately investigated and resolved. The Institutional Review Board approved study procedures. The study was approved by institutional ethic committee of the University Hospitals of Strasbourg.

Open Access Statement: This is an Open Access article distributed in accordance with the Creative Commons Attribution-NonCommercial-NoDerivs 4.0 International License (CC BY-NC-ND 4.0), which permits the non- commercial replication and distribution of the article with the strict proviso that no changes or edits are made and the original work is properly cited (including links to both the formal publication through the relevant DOI and the license). See: https://creativecommons.org/licenses/by-nc-nd/4.0/.

\section{References}

1. Potapov EV, Antonides C, Crespo-Leiro MG, et al. 2019 EACTS Expert Consensus on long-term mechanical circulatory support. Eur J Cardiothorac Surg 2019;56:230-70.

2. Bellavia D, Iacovoni A, Scardulla C, et al. Prediction of right ventricular failure after ventricular assist device implant: systematic review and meta-analysis of observational studies. Eur J Heart Fail 2017;19:926-46.

3. Lampert BC, Teuteberg JJ. Right ventricular failure after left ventricular assist devices. J Heart Lung Transplant 2015;34:1123-30.

4. Moazami N, Fukamachi K, Kobayashi M, et al. Axial and centrifugal continuous-flow rotary pumps: A translation from pump mechanics to clinical practice. J Heart Lung Transplant 2013;32:1-11.

5. Ricklefs M, Hanke JS, Dogan G, et al. Less Invasive Surgical Approaches for Left Ventricular Assist Device Implantation. Semin Thorac Cardiovasc Surg 2018;30:1-6.

6. Schmitto JD, Molitoris U, Haverich A, et al. Implantation of a centrifugal pump as a left ventricular assist device through a novel, minimized approach: upper hemisternotomy combined with anterolateral thoracotomy. J Thorac Cardiovasc Surg 2012;143:511-3.

7. Han J, Pinsino A, Sanchez J, et al. Prognostic value of vasoactive-inotropic score following continuous flow left ventricular assist device implantation. J Heart Lung Transplant 2019;38:930-8.

8. Doenst T, Lamelas J. Do we have enough evidence for minimally-invasive cardiac surgery? A critical review of scientific and non-scientific information. J Cardiovasc Surg (Torino) 2017;58:613-23.

9. Schmitto JD, Mokashi SA, Cohn LH. Minimally-invasive valve surgery. J Am Coll Cardiol 2010;56:455-62.

10. Maltais S, Anwer LA, Tchantchaleishvili V, et al. Left Lateral Thoracotomy for Centrifugal Continuous-Flow Left Ventricular Assist Device Placement: An Analysis from the Mechanical Circulatory Support Research Network. ASAIO J 2018;64:715-20.

11. Pasrija C, Sawan MA, Sorensen E, et al. Less invasive left ventricular assist device implantation may reduce 
right ventricular failure. Interact Cardiovasc Thorac Surg 2019;29:592-8.

12. Ricklefs M, Heimeshoff J, Hanke JS, et al. The influence of less invasive ventricular assist device implantation on renal function. J Thorac Dis 2018;10:S1737-42.

13. Wert L, Chatterjee A, Dogan G, et al. Minimally invasive surgery improves outcome of left ventricular assist device surgery in cardiogenic shock. J Thorac Dis 2018;10:S1696-702.

14. Mohite PN, Sabashnikov A, Raj B, et al. Minimally Invasive Left Ventricular Assist Device Implantation: A Comparative Study: Minimally Invasive Lvad Implantation. Artif Organs 2018;42:1125-31.

15. McGee E Jr, Danter M, Strueber M, et al. Evaluation of a lateral thoracotomy implant approach for a centrifugalflow left ventricular assist device: The LATERAL clinical trial. J Heart Lung Transplant 2019;38:344-51.

16. Hoit BD. Anatomy and Physiology of the Pericardium. Cardiol Clin 2017;35:481-90.

17. Lindqvist $\mathrm{P}$, Holmgren A, Zhao Y, et al. Effect of pericardial repair after aortic valve replacement on

Cite this article as: Carmona A, Hoang Minh T, Perrier S, Schneider C, Marguerite S, Ajob G, Mircea C, Mertes PM, Ramlugun D, Atlan J, Von Hunolstein JJ, Epailly E, Mazzucotelli JP, Kindo M. Minimally invasive surgery for left ventricular assist device implantation is safe and associated with a decreased risk of right ventricular failure. J Thorac Dis 2020;12(4):1496-1506. doi: 10.21037/jtd.2020.02.32 septal and right ventricular function. Int J Cardiol 2012;155:388-93.

18. Rösner A, Avenarius D, Malm S, et al. Changes in Right Ventricular Shape and Deformation Following Coronary Artery Bypass Surgery-Insights from Echocardiography with Strain Rate and Magnetic Resonance Imaging. Echocardiography 2015;32:1809-20.

19. Baxter RD, Tecson KM, Still S, et al. Predictors and impact of right heart failure severity following left ventricular assist device implantation. J Thorac Dis 2019;11:S864-70.

20. de By TMMH, Mohacsi P, Gahl B, et al. The European Registry for Patients with Mechanical Circulatory Support (EUROMACS) of the European Association for Cardio-Thoracic Surgery (EACTS): second report. Eur J Cardiothorac Surg 2018;53:309-16.

21. Sileshi B, Haglund NA, Davis ME, et al. In-hospital outcomes of a minimally invasive off-pump left thoracotomy approach using a centrifugal continuousflow left ventricular assist device. J Heart Lung Transplant 2015;34:107-12. 